\title{
THE DYNAMICS OF MESOSCOPIC NORMAL TUNNEL JUNCTIONS
}

\author{
Kieran MULLEN ${ }^{1}$, Yuval GEFEN ${ }^{2}$ and Eshel BEN-JACOB ${ }^{1,3}$ \\ 'Department of Physics, University of Michigan, Ann Arbor, MI 48109, USA \\ 'Department of Nuclear Physics, The Weitzmann Institute, Rehovot 76100, Israel \\ ${ }^{3}$ School of Physics and Astronomy, Raymond and Beverly Sackler Faculty of Exact Sciences, Tel-Aviv University, \\ 69978 Tel-Aviv, Israel
}

\begin{abstract}
We first review the dynamics of mesoscopic tunnel junctions within the semiclassical approach, outlining the method and its assumptions. We examine the case of a single junction driven by a constant current source, and its non-linear $I-V$ characteristic. We then consider a single junction driven by a direct and an alternating current source, and show that it can be used as a phase-voltage converter. We also apply the approach to two junctions in series, showing that such a configuration has a non-linear $I-V$ characteristic that can be used in electronic devices. We subsequently investigate the dynamics of such systems when the phase breaking time is large and the semiclassical approach does not apply. We first review the standard Zener tunneling calculations, and then show the effect of dissipation. We define and calculate the Zener time, a measure of the width of non-adiabatic transitions. We investigate the dynamics of systems whose energy spectrum consists of a set of periodic bands, for the cases where the phase breaking time is larger and smaller than the period of the band, but larger than the Zener time.
\end{abstract}

\section{Introduction}

Recent advances in the fabrication of submicron systems [1] have made it possible to study experimentally phenomena on mesoscopic length scales. From a theoretical point of view this is of interest since we are dealing with systems that have many degrees of freedom but are not sufficiently large to be in the thermodynamic limit. Yet despite these degrees of freedom, the systems are small enough to demonstrate quantum mechanical dynamics and coherence like those found in microscopic systems [2]. In addition, many questions arise from the coupling of microscopic phenomena to macroscopic objects. For example, since we are generally interested in systems that are coupled to an external driving force, the question of how to describe such open systems within a quantum mechanical framework naturally arises. From the experimental and technological point of view it is hoped that the study of mesoscopic electronic systems will lead to new applications in logical elements and sensitive measurement devices.

In this paper we focus on the recently predicted voltage oscillations in mesoscopic normal tunnel junctions [3-14] with an ultrasmall capacitance $C$, when they are driven by an ideal current source, $I_{\mathrm{dc}}$. Various models predict that the application of a current source to such junctions will produce voltage oscillations with an amplitude of $e / 2 C$, and a frequency $I_{\mathrm{dc}} / e$, similar to the predicted Bloch oscillations of frequency $I_{\mathrm{dc}} d$ $2 e$ in ultrasmall capacitance Josephson junctions [15-21]. One fundamental requirement for the observability of this "inverse Josephson effect" in normal (i.e., not superconducting) junctions, is a large single electron charging energy, $E_{\mathrm{C}}=$ $e^{2} / 2 C$, relative to the average thermal energy $k_{\mathrm{B}} T$. With current technology, junctions with a capacitance $\sim 10^{-17} \mathrm{~F}$ can be produced, which implies a charging energy of $\sim 100 \mathrm{~K}$. For the appropriate experimental conditions the charging energy will dominate the dynamics.

In section 2 we summarize the semiclassical model for normal tunnel junctions, which is described in more detail in refs. [6], [11], and [14]. We outline the method and some of its underlying assumptions, and present the theoretical predictions for the average voltage and the voltage noise. We then consider the special cases of a junction driven by an alternating external force 
$[3,6,11]$, and two junctions connected in series $[6,22,23]$. In the former case we show that mesoscopic junctions can exhibit voltage "spikes" analogous to the Shapiro steps found in Josephson junctions. These steps can be used to construct a direct phase to voltage converter [11]. In the latter case we show that serially coupled junctions can exhibit a staircase $I-V$ characteristic [23], a finding in agreement with experiment [24-26], and that they can be used to form a novel type of transistor $[6,22,27]$. We conclude the section by discussing under what conditions some of the basic assumptions of the model may break down.

In section 3 we go beyond the semiclassical model to consider the effects of quantum coherence on junction dynamics. We argue that nonadiabatic transitions (i.e. Zener tunneling $[28,29])$ may be relevant in this limit and present the results of a few initial steps taken into this complex subject. We show the effects of dissipation on Zener tunneling in the adiabatic limit [30]. We define a new characteristic, the Zener time $\tau_{\mathrm{z}}$, which describes the time interval over which the transition occurs [31]. A similar concept in the context of a time dependent problem was discussed earlier by Landauer and Büttiker [32]. We calculate $\tau_{\mathrm{Z}}$ in the adiabatic and sudden limit, and discuss its relevance to the dynamics of mesoscopic systems.

We conclude in section 4 by discussing possible directions of further research on coherent dynamics, and raise some open questions on the interaction of microscopic and macroscopic systems.

\section{The semiclassical model}

\subsection{Basic review}

The basic view of the semiclassical picture [11] is that the passage of current through a normal tunnel junction arises from a series of stochastic, single electron, tunneling events. That is, if $Q(t)$ denotes the charge across a tunnel junction at time $t$ when it is driven by a current source of magnitude $I_{\mathrm{dc}}$, then the dynamics of the junction can be entirely expressed by a stochastic equation for $Q$ at a later time $t+\Delta t$ :

$$
\begin{aligned}
& Q(t+\Delta t)= \\
& \begin{cases}Q(t)+I_{\mathrm{dc}} \Delta t+e, & \text { with probability } l(Q) \Delta t, \\
Q(t)+I_{\mathrm{dc}} \Delta t-e, & \text { with probability } r(Q) \Delta t, \\
Q(t)+I_{\mathrm{dc}} \Delta t, & \text { with probability } \\
& 1-(r(Q)+l(Q)) \Delta t,\end{cases}
\end{aligned}
$$

where $r(Q)$ and $l(Q)$ are the instantaneous rates of tunneling forward and backward across the junction. If $\tau^{-1}(E)$ is the tunneling rate for an electron in a state of energy $E$, then the net transition rate is calculated by integrating over the single electron energy levels:

$$
\begin{aligned}
r(Q)= & \int_{-\infty}^{\infty} D(E) \tau^{-1}(E) f\left(E-E_{\mathrm{r}}\right) \\
& \times\left(1-f\left(E-E_{\mathrm{l}}^{\prime}\right)\right) \mathrm{d} E \\
l(Q)= & \int_{-\infty}^{\infty} D(E) \tau^{-1}(E) f\left(E-E_{\mathrm{l}}\right) \\
& \times\left(1-f\left(E-E_{\mathrm{r}}^{\prime}\right)\right) \mathrm{d} E
\end{aligned}
$$

where $D(E)$ is the density of states, $f$ is the Fermi distribution function, $E_{\mathrm{r}}$ and $E_{\mathrm{l}}$ are the Fermi levels before an electron tunnels for the right- and left-hand sides, respectively, and $E_{\mathrm{r}}^{\prime}$ and $E_{\mathrm{I}}^{\prime}$ are those after the electron has tunneled. For our purposes we assume that $\tau^{-1}(E)$ and $D(E)$ are energy independent so that $\tau^{-1}(E)=$ $\tau_{0}^{-1}$ and $D(E)=D_{0}$. First we consider the case where the junction is driven by a voltage source, a device which holds the voltage across the junction fixed at a value $V_{\mathrm{dc}}[11]$. Since the voltage is fixed, the tunneling of a single electron across the junction does not alter the relative difference in the Fermi energies on the left- and right-hand sides, so that $E_{1}^{\prime}=E_{\mathrm{r}}+e V_{\mathrm{dc}}=E_{1}-e Q / C$, where $C$ is the junction capacitance. Analysis of eq. (2.1) yields the standard Ohmic result, with $R \equiv$ $1 / e^{2} \tau_{0}^{-1} D_{0}$ [33]. However, if we replace the voltage source by a current source, a device that delivers continuous charge at a fixed rate [11], 
then each electron that tunnels will change the net charge across the junction. In this case, $E_{\mathrm{I}}^{\prime}=E_{\mathrm{r}}-e Q / C+e^{2} / 2 C$, with the result that forward tunneling is suppressed for $Q<e / 2$. Although this effect is negligible for conventional junctions, the small capacitance of mesoscopic junctions $\left(<10^{15} \mathrm{~F}\right)$ makes it practical for the shift in the Fermi levels to be larger than $k_{\mathrm{B}} T$.

From eq. (2.1) we can derive a master equation [34] for the time evolution of $\rho(Q, t)$, the probability density for the charge across the junction to be $Q$ at time $t$ :

$$
\begin{aligned}
\frac{\partial \rho(Q, t)}{\partial t}= & -I_{\mathrm{dc}} \frac{\partial \rho(Q, t)}{\partial Q}+r(Q+e) \rho(Q+e, t) \\
& +l(Q-e) \rho(Q-e, t) \\
& -(r(Q)+l(Q)) \rho(Q, t) .
\end{aligned}
$$

Analysis of eq. (2.3) $[6,35]$ and direct simulation [11] show that for small currents $\left(I_{\mathrm{dc}} \leqslant e / R C\right)$ the junction will charge up to a voltage $\sim e / 2 C$ and then discharge, producing a sawtooth voltage as a function of time with a frequency $I_{\mathrm{dc}} / e$ (fig. 1). At low temperatures $\left(k_{\mathrm{B}} T<e^{2} / 2 C\right)$ the average voltage across the junction is

$$
\langle V\rangle \approx \sqrt{\pi I_{\mathrm{dc}} \operatorname{Re} / 2 C},
$$

and becomes linear for $I_{\mathrm{dc}}>e / R C$, but with a

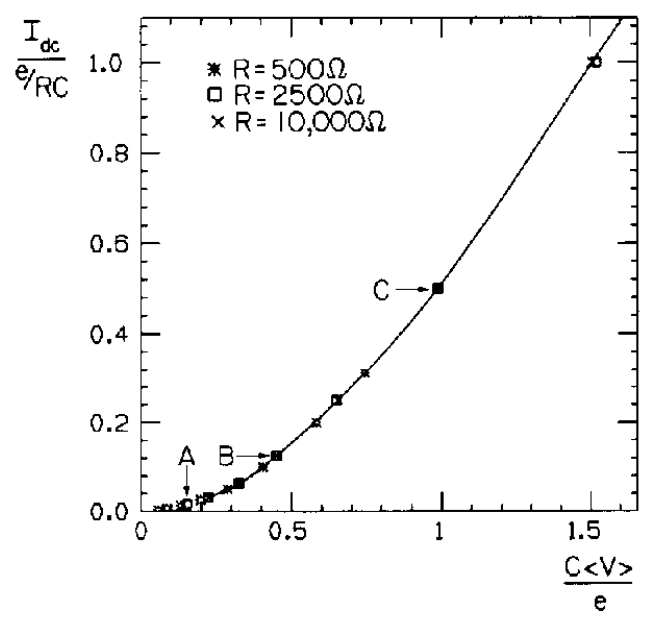

Fig. 1. The $I-V$ characteristic for the semiclassical picture. The parameters are $C=1 \mathrm{fF}$ and $T=0.05 \mathrm{~K}$. voltage offset:

$$
\langle V\rangle \approx I_{\mathrm{dc}} R-\frac{e}{2 C} .
$$

In fig. 2 we show the time dependence of the stochastic process for different values of the current and the corresponding power spectra $[6,11]$. At low currents we can observe the oscillations at a frequency $I_{\mathrm{dc}} / e$. In this limit the background noise in the power spectrum is in accordance with refs. [36] and [37]. Here we are interested in $S_{v}$, the spectral density of the voltage fluctuations. To employ the results of refs. [35] and [36] we have to view the tunnel junction as an RC circuit. Then $S_{V}=|Z|^{2} S_{I}$, where $S_{I}$ is the power spectrum of the current noise and $|Z|^{2}=R^{2} /\left(1+\omega^{2} C^{2} R^{2}\right)$. The $I_{\mathrm{dc}} / e$ oscillations appear in the power spectrum as peaks of zero width at angular frequencies $\omega=2 \pi l l_{\mathrm{dc}} / e$, where $l$ is an integer. The peaks are sharp because the tunneling events have a stationary distribution about a fixed time periodic sequence of period $e / I_{\mathrm{dc}}$. Since the charge can only tunnel in units of $e$, the current source effectively acts as an external clock at frequency $I_{\mathrm{dc}} / e$.

As the current increases the background shot noise also increases, so that when $I_{\mathrm{dc}}>e / R C$ the peaks that correspond to the oscillations are washed out by the noise. In this limit the power spectrum of the current noise approaches that of "classical" shot noise, $S_{I} \approx e I_{\mathrm{dc}}$. The power spectrum of $S_{V}$ has large contributions at low frequencies, as shown in fig. 2(f).

In a small junction $D(E)$ and $\tau^{-1}(E)$ may depend upon energy, but this is not expected to modify the above discussion qualitatively so long as this dependence is smooth. Note, however, that for the case of quasiparticle tunneling between two superconductors, if $\Delta(T)$ is the superconducting energy gap, then $D(E)$ is zero for $\left|E-E_{f}\right|<\Delta(T)$, and diverges as $\left|E-E_{f}\right|$ approaches $\Delta(T)$ from above [33]. The abrupt change in $D(E)$ causes a sharp jump in the transition rates at $Q=2 \Delta(T) C / e+e / 2$. This strong non-linearity is made manifest in the quasiparticle $I-V$ characteristics [20].

Despite the apparent simplicity of the approach, there are several subtle underlying as- 

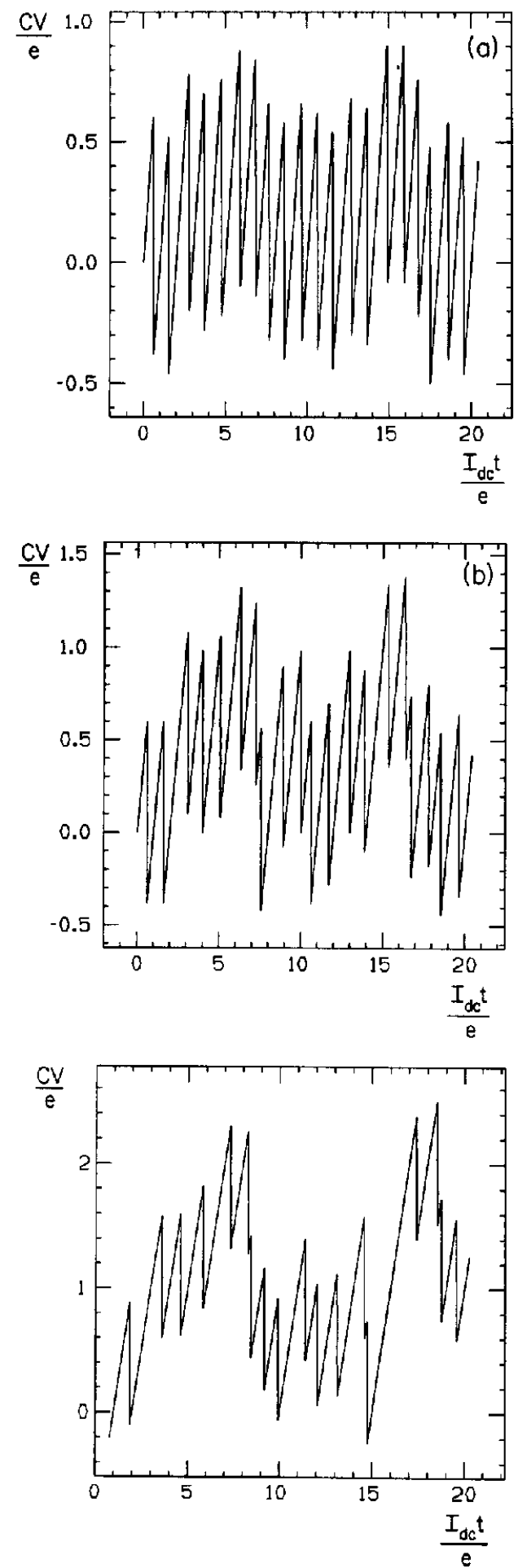

$S(\omega)$

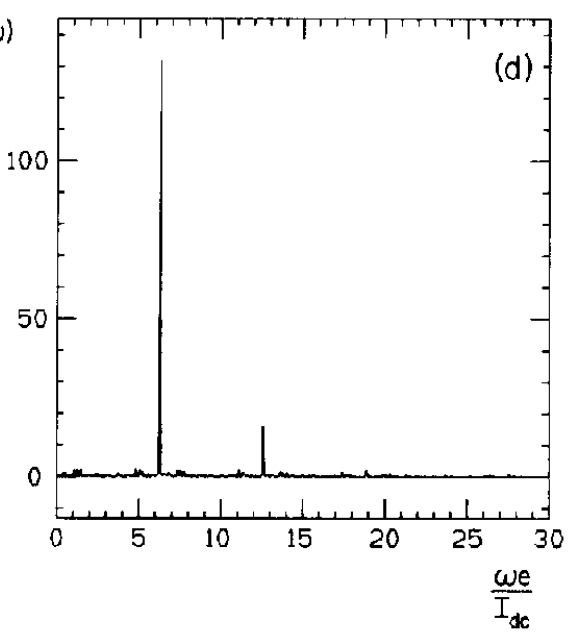

$S(\omega)$

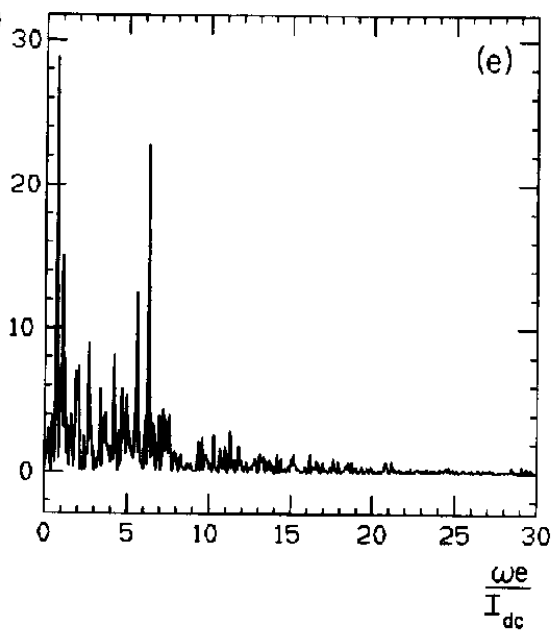

$S(\omega)$

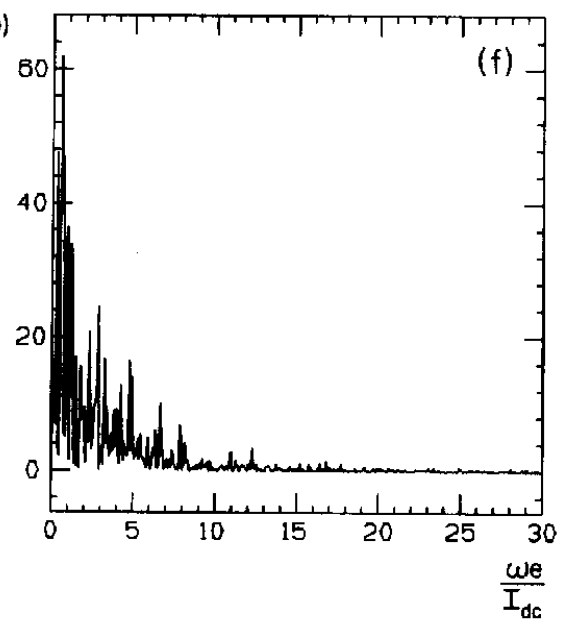

Fig. 2. $V(t)$ as calculated numerically from eqs. (2.1) and (2.2). Processes (a), (b) and (c) correspond to the points A, B and C in fig. 1 and (d), (e) and (f) are the corresponding power spectra. 
sumptions [11] that need firmer microscopic justification, especially in regard to determining the parameter range for which they are valid. In order for the junction to be described by eqs. (2.1) and (2.2) we must at least assume:

(1) The equilibration time in both electrodes is much shorter than the tunneling time $\tau(E)$ so that the occupation probabilities of the states are given by the equilibrium Fermi distribution.

(2) The quantum probabilities for the elastic tunneling of electrons of different states are independent.

(3) The current source can deliver a charge at a continuous and uniform rate so that the fluctuations in $Q$ are much smaller than the electronic charge, $e$.

(4) The resistance of the junction is large enough so that at almost any given moment the electrons are localized on one side of the junction or the other.

(5) The phase randomization time is sufficiently short that coherent multiple tunneling events (cases where a single electron repeatedly tunnels back and forth across the junction) can be neglected.

(6) The capacitance used to calculate the change in the electrostatic charging energy is the same for all single electron states, and is given by the conventional macroscopic capacitance.

We examine the results of relaxing some of these assumptions in section 3 .

\subsection{The effects of an alternating current source}

The voltage oscillations in a single junction have not yet been observed; they are difficult to measure due to their high frequency. Therefore it is natural to examine the case of a mesoscopic normal tunnel junction driven by an oscillating current source as well as one constant in time, in the hope that effects due to the interference of the two frequencies may be visible $[3,6,11]$. In addition, such considerations are important for device applications.

It is possible to generalize eq. (2.1) to the case where the junction is driven by both a direct and an alternating force:
$I=I_{\mathrm{dc}}+I_{\mathrm{ac}} \sin \left(\omega_{\mathrm{ex}} t+\phi\right)$,

where we assumed that $Q=0$ at $t=0$. Drawing an analogy with the behavior of macroscopic Josephson junctions driven by microwaves, we expect to observe resonance phenomena when $\omega_{\mathrm{ex}}$ is commensurate with the angular frequency of the voltage oscillations. That is, when $I \ll$ $e / R C$ so that the voltage oscillations are regular and "well-defined" (as in fig. 2(a)), we expect to see "inverse Shapiro" steps in the response of the system when

$2 \pi \frac{I}{e}=\frac{l}{m} \omega_{\mathrm{ex}}$

where $l$ and $m$ are integers. These steps are different from the standard Shapiro steps. In the latter the steps correspond to regions where the voltage across the junction does not change with the current, so that the step is scanned by varying the current. In the former case the steps should be scanned by holding the amplitudes of the direct and alternating driving force fixed while changing $\phi$, the phase of the alternating force. To demonstrate this we show in fig. 3 the results of numerical calculations of the average voltage across a normal tunnel junction in the

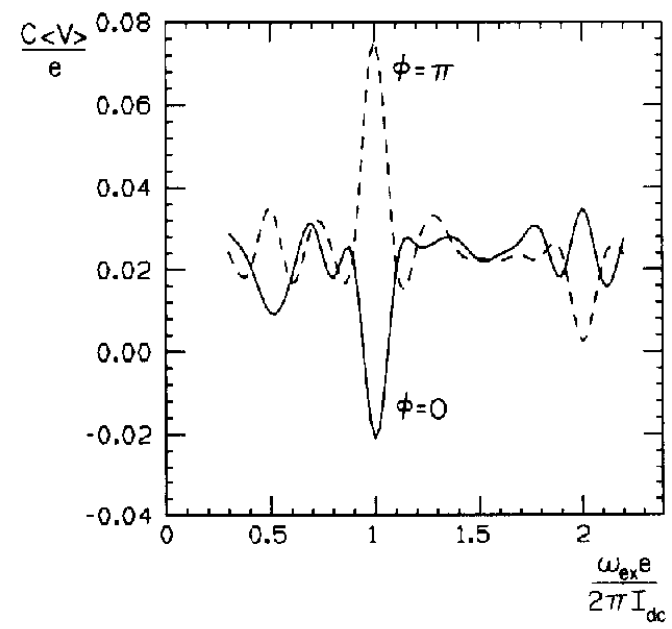

Fig. 3. The average voltage as a function of the external frequency, $\omega_{e x}$, for the semiclassical picture of eq. (2.1) in the presence of a current described by eq. (2.6). The parameters are $R=500 \Omega, C=1 \mathrm{fF}, T=0.05 \mathrm{~K}, I_{\mathrm{dc}}=0.1 \mathrm{nA}$ and $I_{\mathrm{ac}} / I_{\mathrm{dc}}=0.4$. 


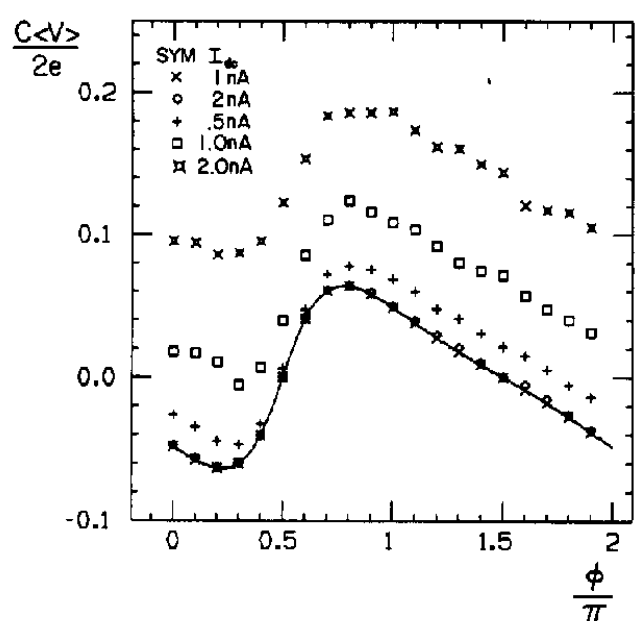

Fig. 4. The average voltage on the first harmonic step $\left(\omega_{e x}=\right.$ $2 \pi I_{\mathrm{dc}} / e$ ), as a function of $\phi$ according to the semiclassical approach. The parameters are $R=2500 \Omega, C=1 \mathrm{fF}$. The solid line shows an analytical approximation [11] for low currents $\left(I_{\mathrm{dc}} \ll e / R C\right)$.

semiclassical picture as a function of $\omega_{\mathrm{ex}}$, for both $\phi=0$ and $\phi=\pi$. In fig. 4 we show the maximal width of the steps when $\phi$ is swept from 0 to $2 \pi$ for selected values of $I_{\mathrm{dc}}$. The first harmonic step $(l=m=1)$ is the largest, and the size of the higher harmonic steps becomes smaller, as in the case of Shapiro steps. Since the average voltage changes as a function of $\phi$, we can use the junction as a phase-voltage converter [11] with maximum sensitivity when $\omega_{\mathrm{ex}}=$ $2 \pi I_{\mathrm{dc}} / e$. The amplitude of the voltage, that is, the size of the first harmonic step, $\Delta V_{1}$, depends upon $I_{\mathrm{dc}} / I_{\mathrm{ac}}$, as shown in fig. 5. This dependence is again very similar to that in the case of "Shapiro steps". In fig. 6 we show the effect of temperature on the average voltage of the first harmonic step as a function of $I_{\mathrm{ac}} / I_{\mathrm{dc}}$.

\subsection{Serially coupled junctions}

The semiclassical model can also be extended to the case of two junctions in series. This configuration is not only important from the standpoint of applications, it is also relevant to understanding the tunneling characteristics of granular materials [22-26, 38, 39]. We consider the case of two junctions in series driven by a voltage

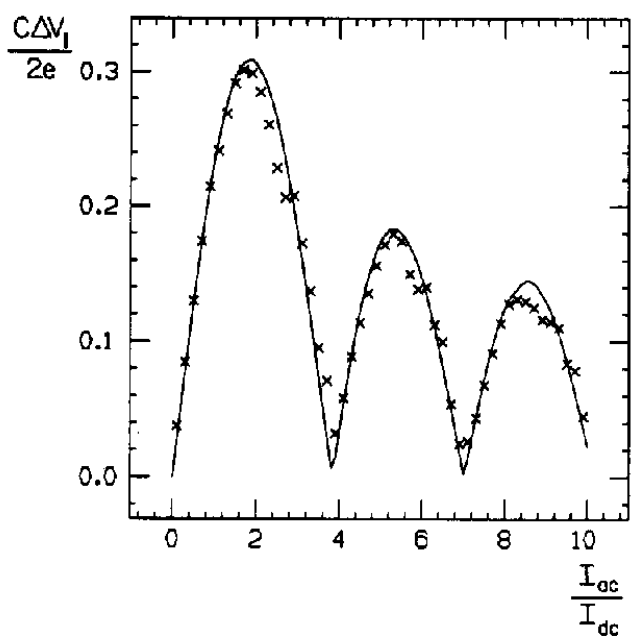

Fig. 5. The maximum width (twice the amplitude) of the first harmonic step as a function of $I_{\mathrm{ac}} / I_{\mathrm{dc}}$. The solid line is an analytical approximation. The parameters are $I_{\mathrm{dc}}=0.1 \mathrm{nA}$, $C=0.05 \mathrm{fF}$ and $R_{\text {in }}=2000 \Omega$.

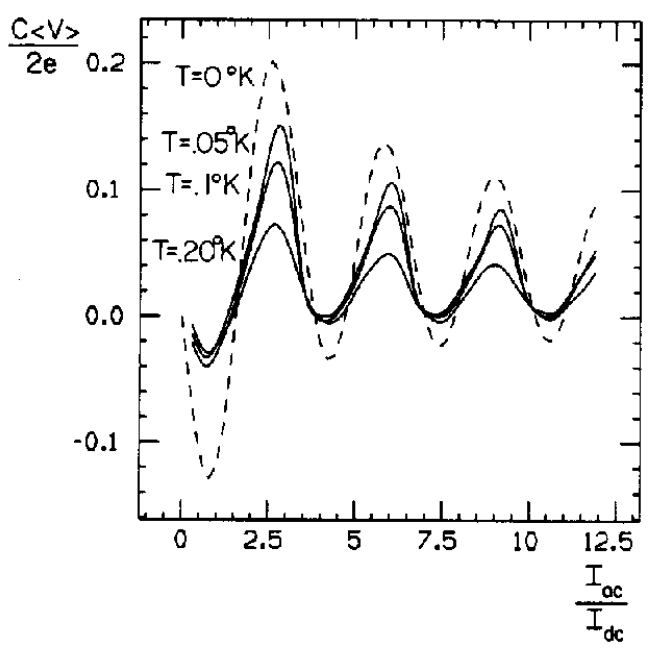

Fig. 6. The average voltage on the first harmonic step $\left(\omega_{\mathrm{ex}}=\right.$ $\left.2 \pi I_{\mathrm{dc}} / \mathrm{e}\right)$ in the semiclassical picture as a function of $I_{\mathrm{ac}} / I_{\mathrm{dc}}$. The parameters are $R_{\mathrm{in}}=2500 \Omega, C=1 \mathrm{fF}, I_{\mathrm{dc}}=0.1 \mathrm{nA}$ and $\phi=0$. The dashed line shows an analytical approximation for $T=0$.

source. The voltage serves to fix the net potential difference across the two junctions at a value $V_{\mathrm{dc}}$, although the voltage across each junction will also depend upon $N$, the number of surplus charges in the interjunction region. A stochastic equation similar to eq. (21) can be used to 
describe the dynamics:

$$
\begin{aligned}
& N(t+\Delta t)= \\
& \begin{cases}N(t)+e, & \text { with probability } \\
N(t)-e, & \left(r_{1}\left(V_{1}\right)+l_{2}\left(V_{2}\right)\right) \Delta t, \\
& \text { with probability } \\
& \left(r_{2}\left(V_{2}\right)+l_{1}\left(V_{1}\right)\right) \Delta t, \\
N(t), & \text { with probability } 1-\left(r_{1}\left(V_{1}\right)+\right. \\
& \left.l_{1}\left(V_{1}\right)+r_{2}\left(V_{2}\right)+l_{2}\left(V_{2}\right)\right) \Delta t,\end{cases}
\end{aligned}
$$

where the subscripts refer to the rates and voltages across the first and second junctions, so that

$$
\begin{aligned}
& V_{1}=V_{\mathrm{dc}} \frac{C_{2}}{C_{1}+C_{2}}-\frac{N e}{C_{1}+C_{2}}, \\
& V_{2}=V_{\mathrm{dc}} \frac{C_{1}}{C_{1}+C_{2}}+\frac{N e}{C_{1}+C_{2}} .
\end{aligned}
$$

As with eq. (2.1), we can write down a master equation for $\rho(N, t)$, the probability that there are $N$ charges between the two junctions at time $t$ :

$$
\begin{aligned}
\frac{\partial \rho(N, t)}{\partial t}= & \left(r_{2}(N+1)+l_{1}(N+1)\right) \rho(N+1, t) \\
& +\left(l_{2}(N-1)+r_{1}(N-1)\right) \rho(N-1, t) \\
& -\left(r_{1}(N)+l_{1}(N)+r_{2}(N)\right. \\
& \left.+l_{2}(N)\right) \rho(N, t),
\end{aligned}
$$

where the transition rates in eq. (2.10) are expressed as functions of $N$. Once the stationary distribution $\rho(N)$ is calculated, the average current can be calculated by summing the current contribution for each $N$, that is $I(N)=r_{2}(N)-$ $l_{2}(N)$, with its proper weight, so that $I=$ $\Sigma_{N}\left(r_{2}(N)-l_{2}(N)\right) \rho(N)$. For an appropriate choice of junction parameters steps will develop in the $I-V$ characteristics (fig. 7). These steps persist even when averaged over an array of junctions whose capacitances and resistances vary by $30 \%$, a prediction in agreement with experiments [24-26].

These results may possibly have important im-

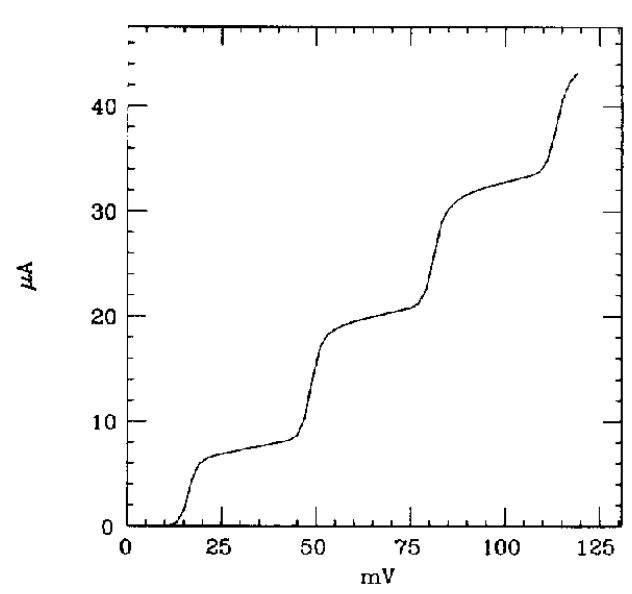

Fig. 7. 1-V characteristic for two ultrasmall capacitance tunnel junctions connected in series driven by an external voltage source, with $C_{1}<C_{2}$ and $R_{1}<R_{2}$. The steps are $e / 2 C_{2}=32 \mathrm{mV}$ wide and have a height $e / R_{2}\left(C_{1}+C_{2}\right)=$ $10.67 \mu \mathrm{A}$. The parameters are $R_{1}=25 \Omega, R_{2}=2500 \Omega, C_{1}=$ $0.001 \mathrm{fF}, C_{2}=0.01 \mathrm{fF}$, and $T=10 \mathrm{~K}$.

plications for tunneling experiments on high $T_{\mathrm{C}}$ superconductors. Tunneling experiments performed on such materials have demonstrated a multiple gap structure in their $\mathrm{d} I / \mathrm{d} V$ curves [40]. We see similar steps in the calculated $I-V$ characteristics of serially coupled superconducting junctions, due to charging effects. If we consider the response of two identical superconducting junctions in series, then there will be a jump in the quasiparticle tunneling current at a voltage of $4 \Delta(T) / e+e / 2 C$, with smaller jumps thereafter at multiples of $e / C$. The high $T_{\mathrm{C}}$ tunneling experiments are generally performed by tunneling from a point contact into a granular superconductor. Since a typical junction formed from the grains is small, the electrostatic charging energy between the tip and a grain, or between grains, is large. When the charging energy and $\Delta(T)$ are comparable for small grain sizes, this charging effect could lead a multiple gap-like $\mathrm{d} I / \mathrm{d} V$ curve [25]. The jumps in the high $T_{\mathrm{C}}$ superconductors would be more rounded, however, due to an averaging over grain size.

Serially connected mesoscopic junctions have valuable device applications as well $[6,23]$. By altering the potential of the interjunction region (say by introducing a third lead or by capacitive coupling) it is possible to increase $N$ by a small 
amount $\delta N$. If $V_{\mathrm{dc}}$ is chosen at a point at the edge of a voltage step, then by varying $\delta N$ we sweep back and forth across the step, producing large changes in the tunneling current [27]. Because the device is small, the characteristic switching time of such a transistor $(\sim R C)$ should be quite short. More pronounced and interesting effects can be seen by making the junctions from a material with a gap at the Fermi energy, so that the transition rates are non-linear.

\subsection{Beyond the semiclassical model}

The semiclassical picture above is expected to give a satisfactory description of the problem when the rate of inelastic interactions is high, so that assumptions (2) and (5) are reasonable. However, for some range of parameters this need not be the case. One may define a dephasing time $\tau_{\phi}$ over which the phase of the electron's wavefunction is lost due to the coupling to an external resevoir (hereafter a single electron picture is implied). On time scales shorter or on the order of $\tau_{\phi}$ a more detailed quantum mechanical treatment is required.

In order to establish the conceptual relation to the Zener tunneling we discuss below, it is useful to consider a simplified picture of two finite ideal metal electrodes. The energy spectrum of each of these electrodes ("left" and "right") when isolated consists of uniformly spaced energy level separated by an energy $\Delta E$. Let us first take the coupling between the left and right electrodes, i.e. the tunneling Hamiltonian, to be zero. Then similarly to what is assumed above in the semiclassical case when this system is connected to an ideal current source, the system can be modeled by shifting the ladder of the single electron energy levels on the left downwards and at the same time shifting the energy levels on the right upwards. Although the energy of the system is quadratic in time, for sufficiently short time scales one can linearize this dependence [12] and obtain two mutually intersecting linear sets of equally spaced levels (fig. 8). The slope of these levels for $t \approx e / 2 I_{\mathrm{dc}}$ (the time for the bias charge to reach $e / 2$ ) is $e I_{\mathrm{dc}} / 2 C$. Now, invoking tunneling between left and right the intersecting levels

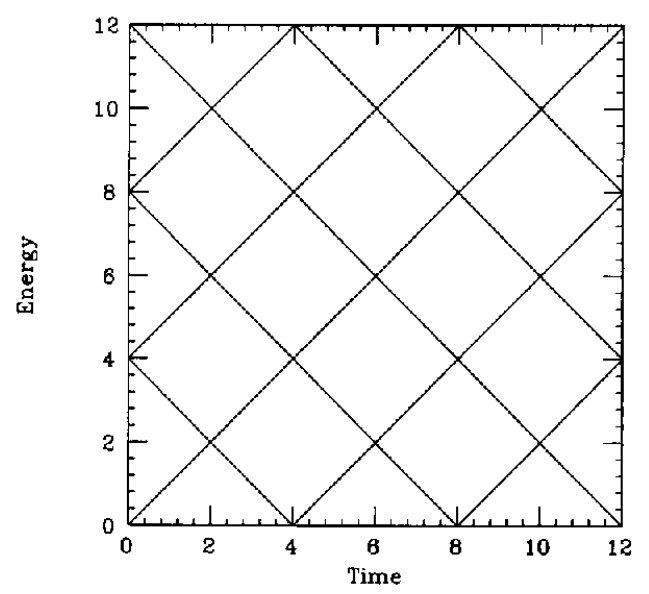

Fig. 8. A schematic description of a current biased junction. The single electron levels are taken to be uniformly spaced. The bias is represented by an upward (downward) shift of the states on the right (left) electrode. This forms a set of crossing single electron bands. The dependence on time has been linearized.

are turned into a set of oscillating bands (fig. 9). A complete quantum mechanical description of such a problem would consist of solving for the dynamics in the energy-time plane, and at the same time account for dissipative processes due to the coupling of the system to an external heat bath. At present this task has not been carried out. However, in the next section we show that several steps towards a comprehensive understanding have been taken.

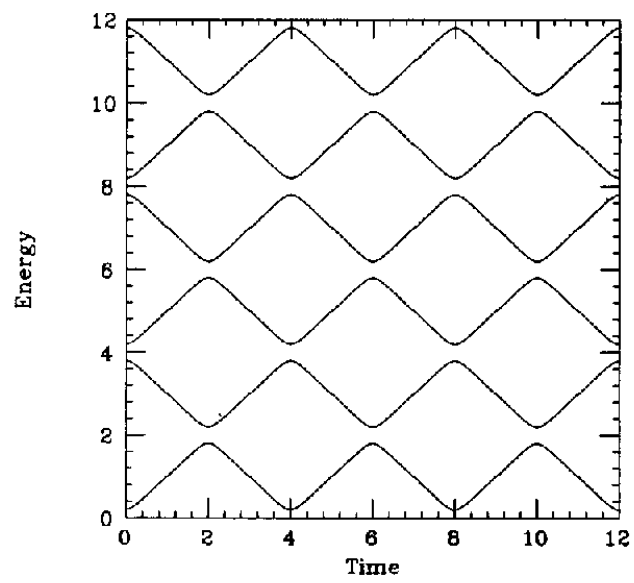

Fig. 9. A schematic description of a current biased junction when the tunneling Hamiltonian is included. The coupling of the different states opens up gaps and forms a set of adiabatic energy microbands. 


\section{Zener tunneling}

\subsection{Basic review}

A variety of systems whose energy depends upon an external parameter (e.g. mesoscopic small normal metal rings in a magnetic field, periodic superlattices in an electric field, and ultrasmall capacitance Josephson junctions driven by a current source), exhibit a periodic microband energy spectrum when this parameter is varied adiabatically in time, as described at the end of the previous section. As discussed above, for some range of parameters the devices may behave coherently and their dynamics cannot be described by a master equation for probabilities, as in eq. (2.3), but must instead be discussed in terms of probability amplitudes. We will attempt to build a partial understanding of these dynamics in successive steps. First we examine Zener transitions in a simple time-dependent two-level Hamiltonian whose asymptotic energy spectrum is linear as a function of time. We will use this simple system to calculate the effect of coupling to a heat bath on the Zener tunneling probability. Next, we define and calculate a "Zener time", the characteristic time it takes for these transitions to occur. We then consider the application of these results to systems with an energy spectrum that consists of a series of bands similar to those found in the "nearly free electron model". Finally, we discuss possible directions for future research.

To gain some insight into the problem we first discuss the simplest model in which Zener tunneling arises, namely that of a two-level timedependent system $[28,29]$. We consider the Hamiltonian:

$H_{\sigma}=\alpha t \sigma_{z}+\Delta \sigma_{x}$,

where the $\sigma$ are the Pauli spin matrices:

$$
\begin{aligned}
& \sigma_{x}=\left(\begin{array}{cc}
0 & 1 \\
1 & 0
\end{array}\right) ; \quad \sigma_{y}=\left(\begin{array}{cc}
0 & -\mathrm{i} \\
\mathrm{i} & 0
\end{array}\right) ; \\
& \sigma_{z}=\left(\begin{array}{cc}
1 & 0 \\
0 & -1
\end{array}\right) .
\end{aligned}
$$

Let us, for the moment, treat time as a parame- ter. For any value of $t$ we can calculate the corresponding eigenvalues of the Hamiltonian (the "adiabatic spectrum"), and the instantaneous eigenstates, as shown in fig. 10. If the system is initially prepared in the lower of these two adiabatic eigenstates (state "down") and this is followed by an adiabatic variation of the Hamiltonian (i.e. in the limit $\hbar \alpha / \Delta^{2} \ll 1$ ), then the system will follow that eigenstate. At any particular time $t_{1}$ the eigenstates of the system , "up" and "down", are orthogonal. However, the state "down" at $t_{1}$ does have an overlap with the state "up" at a later time $t_{2}$. This is the underlying mechanism for Zener tunneling. If we prepare the system in state "down" at $t=-\infty$, then there is a finite probability $P$ that we will find it in the state "up" at $t=\infty$, given by

$P=\exp \left(\frac{-\pi \Delta^{2}}{\hbar \alpha}\right)=\exp \left(\frac{-\pi}{\gamma}\right)$,

where $\gamma \equiv \hbar \alpha / \Delta^{2}$. When $\gamma$ is small the transition is adiabatic; when $\gamma$ is large it is in the sudden limit. Details of the calculation can be found in refs. [28-31].

It is important to note that this result can be generalized to systems whose energy spectrum consists of two intersecting parabolas that both open upward. This can be seen intuitively by

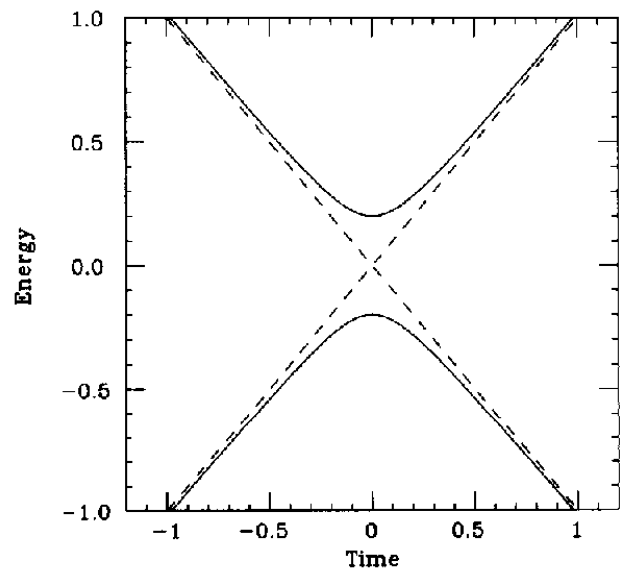

Fig. 10. Energy spectrum of eq. (3.1) with time treated as a parameter. The dashed line corresponds to the case of $\alpha=$ $1.0, \Delta=0$; for the solid line $\Delta>0.2$. The state "up" corresponds to the upper solid line, and the state down refers to the lower solid line. 
noting that the energy difference between the two eigenstates varies linearly in time, so that by factoring out a time-dependent phase from the wavefunctions we can recast the Hamiltonian in the form of eq. (3.1) [31].

\subsection{The effect of dissipation}

The interaction of the system with external degrees of freedom may alter the asymptotic transition probability. We distinguish here between two main effects of coupling to an external heat bath. On the one hand, thermal transitions between the adiabatic energy levels will be induced, corresponding to an absorption or emission of an energy quantum ("phonon") from the bath. A golden rule type expression for the rate of such transitions is derived in appendix $A$ of ref. [30]. On the other hand, coupling to the external heat bath will affect the Zener transitions themselves. To evaluate this latter effect we consider a Hamiltonian of the form:

$H=H_{\sigma}+H_{\mathrm{b}}+H_{\mathrm{c}}$,

where $H_{\mathrm{o}}$ is defined above, $H_{\mathrm{b}}$ is the bath Hamiltonian:

$H_{\mathrm{b}}=\sum_{\beta} \hbar \omega_{\beta}\left(a_{\beta}^{\dagger} a_{\beta}+\frac{1}{2}\right)$

and $H_{\mathrm{c}}$ is the coupling between the two:

$H_{\mathrm{c}}=\sum_{\beta} \sigma_{z} A_{\beta}\left(a_{\beta}+a_{\beta}^{\dagger}\right)$.

Here $a_{\beta}\left(a_{\beta}^{\dagger}\right)$ is a Bosonic annihilation (creation) operator of the mode $\beta$, and $A_{\beta}$ is the strength of the coupling to mode $\beta$. A detailed calculation for the adiabatic case in which it is assumed that $\Delta \sigma_{x}$ is a perturbation and $k_{\mathrm{B}} T \ll 2 \Delta$, is presented in ref. [30]. The modified Zener tunneling probability as a function of $T$ is plotted in fig. 11 for several values of $\eta$, where

$\eta \equiv \sum_{\beta} \frac{A_{\beta}^{2}}{\omega_{\beta}^{2}} \delta\left(\omega-\omega_{\beta}\right)$

From fig. 11 we can see that the Zener tunneling

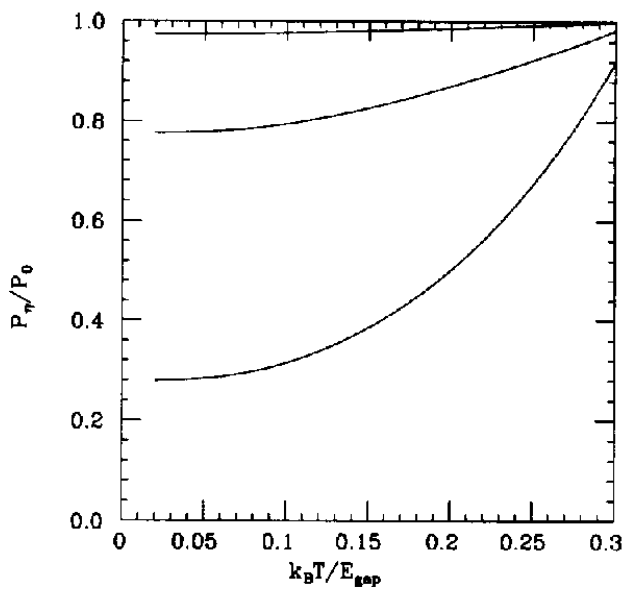

Fig. 11. A plot of the ratio of the Zener transition probability with dissipation to the bare Zener transition probability (eq. (3.3)) as a function of temperature for different values of the dissipation $\eta$. The current is chosen such that $\gamma=0.2$. The parameters are $\eta=0.1$ (top curve), $\eta=1.0$ (middle curve) and $\eta=10$ (bottom curve).

probability is enhanced as temperature is increased, due to phonon assisted tunneling, and is suppressed as $\eta$ is increased. The latter can be understood in view of the fact that the Zener transition amplitude is a manifestation of the overlap between an initial "up" state and a final "down" state. As we increase the accessibility to larger parts of Hilbert space this overlap will, in general, diminish.

\subsection{Zener time}

Not only is it valuable to know the asymptotic Zener transition probability, it is important to know its time dependence as well. Knowing this time dependence is valuable for incoporating interference effects among consecutive Zener transitions and accounting for inelastic effects. For example, if the dephasing time $\tau_{\phi}$ is longer than the characteristic time for Zener tunneling, $\tau_{\mathrm{z}}$, then the transition probability of eq. (3.3) will be valid. If $\tau_{\phi}<\tau_{\mathrm{z}}$, then the effects of dissipation must be considered. In addition, it is important to know $\tau_{\mathbf{Z}}$ when the energy spectrum of the system forms a set of periodic bands as a function of an external parameter (fig. 9). In this case when $\tau_{\mathrm{Z}}$ is much smaller than the period of 
the spectrum the complex system may be viewed as a succession of simple two-level systems.

It is possible to solve for the probability of being in state "up" as a function of time, by solving eq. (3.1) exactly. Following Zener [28] we may write eq. (3.1) as a pair of coupled differential equations:

$\mathrm{i} \hbar \frac{\partial \psi_{1}}{\partial t}=\alpha t \psi_{1}+\Delta \psi_{2}$

$\mathrm{i} \hbar \frac{\partial \psi_{2}}{\partial t}=-\alpha t \psi_{2}+\Delta \psi_{1}$

where $\psi_{1}$ and $\psi_{2}$ are the full time-dependent solutions of eq. (3.1), not the "up" and "down" states. The solution for this system can be expressed in terms of parabolic cylinder functions, or can be solved numerically for a given set of initial conditions; an example of the latter is shown in fig. 12. By examining the width over which the transition occurs, measuring the time from the center of the transition to a point where it has developed half of its asymptotic value, a characteristic "Zener time", $\tau_{\mathrm{z}}$, can be defined. We stress that the "Zener time" is only one of the relevant parameters necessary in any attempt to understand the effect of dissipation on such a system.

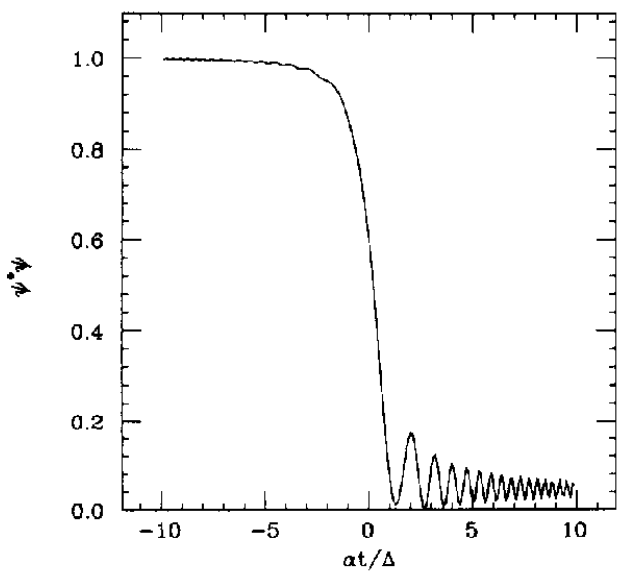

Fig. 12. A typical numerical solution of eq. (3.8), with $\gamma=1.0$. The initial conditions were $\psi_{1}=1$ and $\psi_{2}=0$ at $t=-100 \Delta / \alpha$. Note that the transition probability $\left(\psi_{1}^{*} \psi_{1}\right)$ and not the amplitude is plotted. The asymptotic transition probability is $\sim 0.04$.
From the parameters of the problem, three natural times can be defined:

$\tau_{\mathrm{C}}=\hbar / \Delta ; \quad \tau_{\mathrm{P}}=\sqrt{\hbar / \alpha} ; \quad \tau_{\mathrm{g}}=\delta / \alpha$.

The time $\tau_{\mathrm{C}}$ is the inverse of the oscillation frequency of a particle in a symmetric double well when the splitting between the symmetric and antisymmetric states is $\Delta ; \tau_{\mathrm{p}}$ is approximately the time it takes for the phase of $\psi$ to change by $2 \pi$ near $t=0$; and $\tau_{\mathrm{g}}$ is the time that it takes the external driving force to transfer an energy $\Delta$ (half the gap) to the system. It is reasonable to expect that in the adiabatic limit $(\gamma \ll 1)$ the time of Zener tunneling must depend on both the coupling and the sweep rate so that $\tau_{\mathrm{z}}=\tau_{\mathrm{g}}$. Since we know that the asymptotic probability is exponentially small in $1 / \gamma$ (from eq. (3.3)), the problem can be analyzed by the WKB method. Such an expression yields a fixed transition profile in units of $\tau_{\mathrm{g}}$, in excellent agreement with direct numerical solutions of eq. (3.8). In the sudden limit $(\gamma \gg 1)$ the system sweeps through the transition region so rapidly that the transition width should be independent of $\Delta$, so that $\tau_{\mathrm{Z}}=$ $\tau_{\mathrm{P}}$. In this limit from eq. (3.3) we expect $P \approx$ $1-\pi / \gamma$, so that $\psi$ can be expanded in a power series in $\gamma^{-1 / 2}$. Both analytic and numerical solutions show that $\tau_{\mathrm{z}}=\tau_{\mathrm{p}}$.

It is possible to define the Zener time in other ways. One alternative method ${ }^{31}$ is to apply an oscillating perturbation and determine $\tau_{\mathrm{z}}$ by examining the frequency dependence of the tunneling probability. This method is similar to one first introduced by Landauer and Büttiker to discuss the tunneling of electrons through real space barriers and through the forbidden gap in the presence of an electric field [32]. Such an approach might indicate the time scale over which the system is sensitive to perturbations.

\subsection{Effects of coherence on junction dynamics}

The results derived above have broad applications. As pointed out at the beginning of this section, many systems have an energy spectrum of periodic bands similar to the one described at the end of section 2 . If we define $\tau_{\mathrm{b}}$ to be the 
time it takes for the system to sweep through one period of the energy bands $\left(\tau_{\mathrm{b}}=e / I_{\mathrm{dc}}\right.$ in the case of normal tunnel junction) then when $\tau_{\mathrm{Z}}<\tau_{\phi} \leqslant$ $\tau_{b}$ such a system can undergo Zener transitions as it passes through regions where the bands pull together, with a probability given by eq. (3.3). The dynamics can then be described by a classical random walk, where the hopping probability depends upon the band number and the derivative of the energy with respect to the driving force. Analysis of such systems has shown that this picture is incomplete and that a random walk with just Zener transitions leads to a steady average drift upward in band number [11].

Clearly, this unbounded drift is not physical. As we continue to pump energy into the system its average energy increases. The system can dissipate this energy by means of inelastic transitions among the bands, thus balancing the drift. Such downward transitions can occur even when the temperature of the coupled heat bath is zero. At non-zero temperatures there is also the finite probability for the system to absorb a phonon from the bath, thus undergoing a transition upward. However, as long as $k_{\mathrm{B}} T$ is smaller than the energy gap between bands, such transitions may be neglected.

The dynamics of the system are then determined by the interplay of coherent and inelastic transitions [11]. If the driving force is small then we can focus on the lowest two bands since the system will make an inelastic transition downward before it can Zener tunnel from the second to the third band. The net effect of both inelastic and Zener transitions can be approximated considering these two processes independently. The voltage (in the case of a tunnel junction) is approximately equal to the voltage calculated with $P=1$, multiplied by the Zener probability of making a transition from the first to the second band, leading to exponentially small dissipation for low values of the driving force. For larger values of the driving force the probability of Zener tunneling is nearly unity, and the response asymptotically approaches that of the $P=1$ case.

Finally, when $\tau_{\phi}>\tau_{\mathrm{b}}$ a more complete quantum mechanical treatment is required. Each period of the band can be treated as a separate, two-level Zener tunneling problem, as discussed earlier in this section. Using the analytic expansion for $\psi$ sketched above, one can calculate the final state amplitudes as a function of the initial state amplitudes. The system can then be analyzed via transfer matrix techniques.

\section{Future directions}

The semiclassical model is tractable because it assumes that the junction dynamics are classical, i.e. they are determined by probabilities, not probability amplitudes. As $\tau_{\phi}$ increases, this approach becomes less appropriate. It is still not known how to describe the junction when it acts coherently for long intervals that are punctuated by inelastic phase breaking collisions. In such a limit we may find novel coherent effects that will alter junction behavior. For example, when $\tau_{\phi}$ grows longer than one period of the band, we expect to see interference effects among the bands. Also, if the junction resistance is less than $\hbar / e^{2}$, the electron wavefunction will extend over both electrodes which may lower the effect of the charging energy to the point where energy modulation due to the charging energy may be too small to be observed. As the junction is made smaller, the density of states will no longer be continuous. This will be expecially important for the case of the serially connected junctions, since the interjunction region can be as small as $50 \AA$. In addition, it may be possible to tunnel to virtual states within the interjunction region, tunneling coherently through the device at voltages where the semiclassical model predicts no current should flow [41].

More generally, how do we understand the dependence of microscopic dynamics on macroscopic variables? For, although these junctions are extremely small, they are made up of a large number of particles, when compared to a single electron. What does it mean to say that a tunneling electron is blocked by an electrostatic interaction with the entire junction? How can this interaction depend upon the geometrical capaci- 
tance, a quantity that is a classical concept? Is it possible to talk about a constant current source on such a level, or will any source produce fluctuations in the charge it delivers on the order of $e$ [42]? All of our classical concepts of electronics must be re-examined upon entering the mesoscopic domain.

\section{Acknowledgements}

One of us (Y.G.) thanks the conference for its hospitality. We have profited from many useful discussions with S. Fishman, R.C. Jaklevic, Z. Schuss, D. Thouless, and M. Amman. We thank R. Landauer for extensive discussions and correspondence. We are grateful to K.K. Likharev for bringing to our attention several works on mesoscopic junctions. This research was partially supported by NSF Grant DMR 8608305, Grant DAAL 03-87-k-0007 from the Army Research Office, a grant from the Israel Academy of Sciences, the Tel Aviv grant for Basic Research, and a grant from the U.S.-Israel B.S.F. Computer simulations were done in part using time granted by the NSF Computer Center on the San Diego Super Computer. One of us (K.M.) was supported by the Center for High Frequency Micro-electronics at the University of Michigan. E.B.-J. and Y.G. are partially supported by the Bat-Sheva foundation.

\section{References}

[1] B.R. Brewer, ed, Electron-Beam Lithography in Microelectronics Fabrication (Academic, New York, 1980).

[2] For a review of mesoscopic systems, see Y. Imry, in: Memorial Volume in Honor of Prof. Shang-Keng Ma, G. Grinstein and G. Mazenko, eds. (World Scientific, Singapore, 1986), and E. Ben-Jacob, M. Büttiker, R.A. Webb, Phys. Today, to be published.

[3] E. Ben-Jacob and Y. Gefen, Phys. Lett. A 108 (1985) 289.

[4] E. Ben-Jacob, Y. Gefen, K. Mullen and Z. Schuss, in: "SQUID 85", H.D. Hahlbohm and H. Lübbig, eds. (Walter de Gruyter, Berlin, 1985).

[5] D.V. Averin and K.K. Likharev, in: "SQUID 85", H.D.
Hahlbohm and H. Lübbig, eds. (Walter de Gruyter, Berlin, 1985).

[6] D.V. Averin and K.K. Likharev, Low Temp. Phys. 62 (1986) 345.

[7] D.V. Averin and K.K. Likharev, Sov. Phys. JETP 63 (1986) 427.

[8] F. Guinea and G. Schön, Europhys. Lett. 1 (1986) 585.

[9] K.K. Likharev, IEEE Trans. Magn. 23 (1987) 1138.

[10] D.V. Averin, Sov. J. Low Temp. Phys. 13 (1987) 208.

[11] E. Ben-Jacob, Y. Gefen, K. Mullen and Z. Schuss, Phys. Rev. B37 (1988) 7400.

[12] Y. Gefen and D.J. Thouless, unpublished.

[13] M. Büttiker, Phys. Rev. B36 (1987) 3548.

[14] K.K. Likharev, preprint.

[15] A. Widom, G. Magaloudis, T.D. Clark, H. Prance and R.J. Prance, J. Phys. A15 (1982) 3877; R.J. Prance, J.E. Motton, H. Prance, T.D. Clark, A. Widom and G. Megalaudis, Helv. Phys. Acta 56 (1983).

[16] T.P. Spiller, J.E. Mutton, H. Prance, R.J. Prance and T.D. Clark, in: "SQUID 85", H.D. Hahlbohm and H. Lübbig, eds. (Walter de Gruyter, Berlin, 1985).

[17] A.l. Larkin, K.K. Likharev and Yr.N. Ouchinnikov, Physica B 126 (1984) 414.

[18] K.K. Likharev and A.B. Zorin, J. Low Temp. Phys. 59 (1985) 347.

[19] W. Zwerger, Phys. Rev. B35 (1987) 4737.

[20] K. Mullen, E. Ben-Jacob and Z. Schuss, Phys. Rev. Lett. 60 (1988) 1097.

[21] K.K. Likharev, Dynamics of Josephson Junctions and Circuits (Gordon and Breach, New York, 1987).

[22] I.O. Kulik and R.I. Sheckter, Sov. Phys. JETP 41 (1975) 308.

[23] K. Mullen, E. Ben-Jacob, R.C. Jaklevic and Z. Schuss, Phys. Rev. B36 (1987) 98.

[24] J.B. Barner and S.T. Ruggiero, Phys. Rev. Lett. 59 (1987) 807.

[25] J.B. Barner and S.T. Ruggiero, Rap. Commun. (1987) 8870 .

[26] L.S. Kuz'min and K.K. Likharev, JETP Lett. 45 (1987) 389.

[27] M. Amman, K. Mullen and E. Ben-Jacob, to be published.

[28] G. Zener, Proc. Roy. Soc. A137 (1932) 696.

[29] G. Wannier, Physics 1 (1965) 251.

[30] Y. Gefen, E. Ben-Jacob and A.O. Caldeira, Phys. Rev. B36 (1987) 2770.

[31] K. Mullen, E. Ben-Jacob, Y. Gefen and Z. Schuss, to be published.

[32] R. Landauer and M. Büttiker, IBJ J. Res. Dev. 30 (1986) 451; Festkorperprobleme 25 (1985) 711.

[33] Tunneling in Solids, E. Burstein and S. Lundqvist, eds. (Plenum, New York, 1968).

[34] N.C. Van Kampen, Stochastic Processes in Physics and Chemistry (North-Holland, Amsterdam, 1981).

[35] E. Ben-Jacob, D.J. Bergman, B.J. Matkowsky and Z. Schuss, Phys. Rev. B34 (1986) 1572.

[36] T.L. Ho, Phys. Rev. Lett. 51 (1983) 2060. 
[37] E. Ben-Jacob, E. Mottola and G. Schön, Phys. Rev. Lett. 51 (1983) 2064.

[38] I. Giaver and H.R. Zeller, Phys. Rev. Lett. 20 (1968) 1504.

[39] J. Lambe and R.C. Jaklevic, Phys. Rev. Lett. 22 (1969) 1371.
[40] I. Iguchi, H. Watanabe, Y. Kasai, T. Mochiku, A. Sugishita and E. Yamaka, Jpn. J. Appl. Phys. 26 (1987) L645.

[41] T.A. Fulton and G.J. Dolan, private communication. [42] A. Legget, private communication. 